Allison Nitsch, MD

ACUTE Center for Eating Disorders at Denver Health, Denver, CO; Department of Medicine, University of Colorado School of Medicine,

Aurora, CO
Heather Dlugosz, MD

Eating Recovery Center and Pathlight Mood \& Anxiety Center, Cincinnati, $\mathrm{OH}$
Dennis Gibson, MD

ACUTE Center for Eating Disorders at Denver Health, Denver, CO; Department of Medicine, University of Colorado School of Medicine, Aurora, CO
Philip S. Mehler, MD, FACP, FAED

ACUTE Center for Eating Disorders at Denver Health, Denver, CO; Department of Medicine, University of Colorado School of Medicine,

Aurora, CO

\title{
Medical complications of bulimia nervosa
}

\section{ABSTRACT}

Bulimia nervosa, a mental illness 4 times more common than anorexia nervosa, is characterized by binge-eating followed by compensatory purging behaviors, which include self-induced vomiting, diuretic abuse, laxative abuse, and misuse of insulin. Patients with bulimia nervosa are at risk of developing medical complications that affect all body systems, especially the renal and electrolyte systems. Behavior cessation can reverse some, but not all, medical complications.

\section{KEY POINTS}

Most people with bulimia nervosa are young and of normal weight, or even overweight, making detection and diagnosis difficult.

As a consequence of purging behaviors, pseudo-Bartter syndrome can develop due to chronic dehydration, placing patients at risk for electrolyte abnormalities such as hypokalemia, as well as marked and rapid edema formation when purging is interrupted.

Electrolyte and metabolic disturbances are the most common causes of morbidity and mortality in patients with bulimia nervosa. Hypokalemia should be managed aggressively to prevent electrocardiographic changes and arrhythmias such as torsades de pointes.

Diabetic patients who purge calories through manipulation of their blood glucose are at high risk for hyperglycemia, ketoacidosis, and premature microvascular complications.

Gastrointestinal complaints are common and include gastroesophageal reflux disease.

doi:10.3949/ccjm.88a.20168

\begin{abstract}
2I-Year-old woman with a history of deA pression and anxiety presents to your clinic for follow-up after an emergency room visit, where she had presented 2 days earlier for feeling like she was going "to pass out" during her college cross-country meet. At the emergency room, the patient was noted to have a serum potassium level of $2.9 \mathrm{mmol} / \mathrm{L}$ (reference range $3.7-5.1 \mathrm{mmol} / \mathrm{L}$ ), bicarbonate $35 \mathrm{mmol} / \mathrm{L}$ ( $22-$ $30 \mathrm{mmol} / \mathrm{L}$ ), and orthostatic hypotension. She was given $2 \mathrm{~L}$ of intravenous normal saline and intravenous and oral potassium.

On follow-up, her vital signs are normal. Her body mass index is $24.5 \mathrm{~kg} / \mathrm{m}^{2}$. She reports feeling better but has noted marked swelling of both her lower extremities, which is causing her distress. The examination is notable for bilateral $2+$ pitting edema and calluses on the dorsal aspect of her right hand.
\end{abstract}

\section{A SERIOUS MENTAL ILLNESS WITH PHYSICAL CONSEQUENCES}

Bulimia nervosa $(\mathrm{BN})$ is a serious mental illness characterized by binge-eating followed by compensatory purging behaviors. It is frequently accompanied by medical sequelae that affect normal physiologic functioning and contribute to increased morbidity and mortality rates. ${ }^{1}$ Most people with BN are of normal weight or even overweight, ${ }^{2}$ and are otherwise often able to avoid detection of their eating disorder. Thus, it is important that clinicians familiarize themselves with these complications and how to identify patients with disordered eating patterns.

Recurrent binge-eating followed by purging $\mathrm{BN}$ is characterized by overvaluation of body weight and shape and recurrent binge-eating 
(consuming an excessive caloric amount in a short period of time, usually a 2 -hour period, that the patient feels unable to control). This is soon accompanied by compensatory purging behaviors that can include abuse of laxatives and diuretics, withholding insulin (termed diabulimia or eating disorder-diabetes mellitus type 1 ), self-induced vomiting, fasting, and excessive exercise. Some patients also abuse caffeine or prescription stimulant medications commonly used to treat attention-deficit/hyperactivity disorder.

Self-induced vomiting and laxative misuse account for more than $90 \%$ of purging behaviors in BN. ${ }^{3}$ The Diagnostic and Statistical Manual of Mental Disorders, Fifth Edition (DSM-5) requires episodes of bingeing and compensatory behaviors in $\mathrm{BN}$ to occur at least once per week over the course of 3 months and not occur during an episode of anorexia nervosa. The complications of purging behaviors found in $\mathrm{BN}$ are identical to those found in the binge-purge subtype of anorexia nervosa except that restriction of calories primarily and excessive weight loss are not present.

The severity of $\mathrm{BN}$ is determined by the frequency of the mode of the purging behaviors (mild: an average of 1-3 episodes of inappropriate compensatory behaviors weekly; moderate: $4-7$; severe: $8-13$; extreme: 14 or more) or the degree of functional impairment. ${ }^{2}$ Some patients may vomit multiple times per day while others may use significant amounts of laxatives. Some may engage in multiple different purging behaviors, which has been shown to be associated with a greater severity of illness. ${ }^{4}$ Exercise is considered excessive if it interferes with other activities, persists despite injury or medical complications, or occurs at inappropriate times or situations. ${ }^{2,5}$

\section{ONSET IN ADOLESCENCE, AND FAIRLY COMMON}

BN typically develops in adolescence or young adulthood and affects both sexes, although it is much more common in girls and young women. ${ }^{6}$ It affects people regardless of sexual orientation but has been shown to be more prevalent in nonheterosexual males. ${ }^{7}$ Studies have found similar prevalence of $\mathrm{BN}$ among different racial and ethnic groups. Individu- als with $\mathrm{BN}$ are generally within or above the normal weight range. ${ }^{2}$

According to pooled data from the World Health Organization, the lifetime prevalence of BN in adults is $1.0 \%$ using the older DSM-IV criteria, ${ }^{7}$ which is greater than the reported prevalence of anorexia nervosa. Prevalence estimates are higher with the broadened DSM- 5 criteria, ranging from $4 \%$ to $6.7 \% .^{8}$

There are multiple predisposing and perpetuating factors-genetic, environmental, psychosocial, neurobiological, and temperamental. These can include impulsivity, developmental transitions such as puberty, internalization of the thin ideal, and weight and shape concerns. ${ }^{9}$ A history of childhood trauma, including sexual, physical or emotional trauma, has also been associated with BN. ${ }^{10}$

More than $70 \%$ of people with eating disorders report concomitant psychiatric comorbidity-affective disorders, anxiety, substance use, and personality disorders are most common in BN. ${ }^{11}$ Psychiatric comorbidities as well as hopelessness, shame, and impulsivity associated with the illness may contribute to challenges with nonsuicidal self-harm, suicidal ideation, and death by suicide. Individuals with $\mathrm{BN}$ experience lifetime rates of nonsuicidal self-harm of $33 \%$ and are nearly 8 times more likely to die by suicide than the general population. ${ }^{12,13}$ The reported standardized mortality rates in those with $\mathrm{BN}$ are less than in those with anorexia nervosa but are still significantly elevated at $1.5 \%$ to $2.5 \%{ }^{1}$

\section{MEDICAL COMPLICATIONS}

As noted earlier, BN is associated with a significantly increased mortality rate even though many of these patients are young. Much of this elevated mortality is attributable to the medical complications associated with $\mathrm{BN}$, which are a direct result of the mode and frequency of purging behaviors. Thus, for example, if someone uses laxatives 3 times per day or vomits 1 time per day, there may be no medical complications, but many patients engage in their respective purging behaviors many times per day, leading to multiple complications. 
Aside from the electrolyte aberrations from purging, some of the medical complications are unique to the mode of purging. Furthermore, $\mathrm{BN}$ has been found to increase the risk of any cardiovascular disease, including ischemic heart disease and death in females. ${ }^{14}$ These same complications may also apply to patients with anorexia nervosa of the bingepurge subtype in contrast to those patients with anorexia nervosa who only restrict caloric intake but do not purge.

We will now discuss, in a systems-based approach, the medical complications that develop in people with $\mathrm{BN}$ as a direct result of their purging behaviors.

\section{Skin}

Russell sign (Figure 1), named after Dr. Gerald Russell, who first defined the disease $\mathrm{BN}$ in 1979, refers to the development of calluses on the dorsal aspect of the dominant hand..$^{15}$ It is pathognomonic for self-induced vomiting and is due to traumatic irritation of the hand by the teeth, from repeated insertion of the hand into the mouth to provoke vomiting. ${ }^{15}$

Russell sign is not commonly seen since many of these patients are able to spontaneously vomit or they utilize utensils to initiate self-induced vomiting.

\section{Teeth and mouth}

Abnormalities of the teeth and mouth, specific for purging via vomiting, include dental erosions and trauma to the oral mucosa and pharynx. ${ }^{16}$

Dental erosion is the most common oral manifestation of chronic regurgitation. It is believed to be caused by the teeth coming into contact with acidic vomitus $(\mathrm{pH} 3.8)$, although just how changes in salivary composition and dietary intake contribute is unclear. It tends to affect the lingual surfaces of the maxillary teeth and is known as perimyolysis. Vomiting also potentially increases the risk of dental caries.

Trauma to the oral mucosa, especially the pharynx and soft palate, is also encountered and is presumed to occur either as a result of the patient inserting a foreign object into the mouth to induce vomiting or the caustic effect of the vomitus on the mucosal lining.

Dental erosions are irreversible once they have developed. Use of fluorinated mouth-

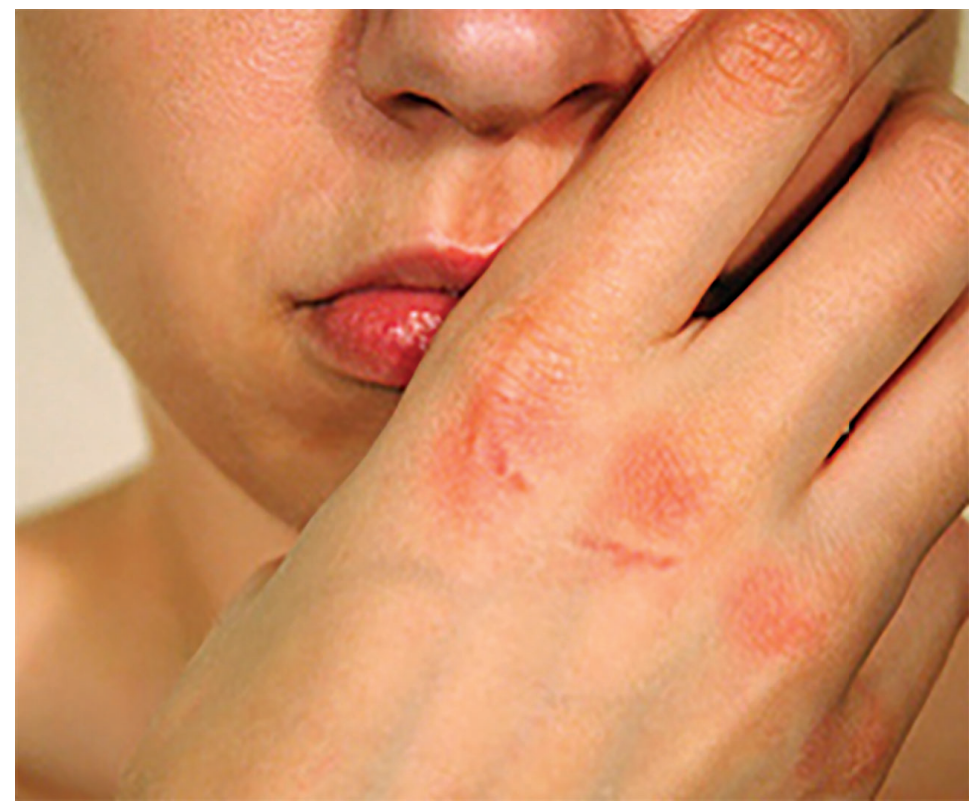

Figure 1. The Russell sign.

wash after purging and horizontal gentle brushing are recommended. Ongoing selfinduced vomiting will also damage newly implanted teeth as well as dental prosthetics.

\section{Head, ears, nose, and throat}

Purging by vomiting increases the risk of subconjunctival hemorrhages from forceful retching, which can also cause recurrent epistaxis. Indeed, recurrent bouts of epistaxis that remain unexplained should prompt a search for covert BN.

Pharyngitis is often noted in those who vomit frequently, due to contact of the pharyngeal tissue with stomach acid. Hoarseness, cough, and dysphagia may also similarly develop. Pharyngeal and laryngeal complaints can be improved with cessation of vomiting and the use of medications to suppress acid production, such as proton pump inhibitors.

\section{Parotid glands}

Parotid gland hypertrophy, or sialadenosis (Figure 2), may develop in more than 50\% of people engaging in purging via self-induced vomiting. ${ }^{17}$ Ironically, it usually develops 3 to 4 days after cessation of purging. Symptoms include bilateral painless enlargement of the parotid glands and, occasionally, other salivary glands. It is believed to develop due to ei-
Dental erosion is the most common oral manifestation due to chronic regurgitation 


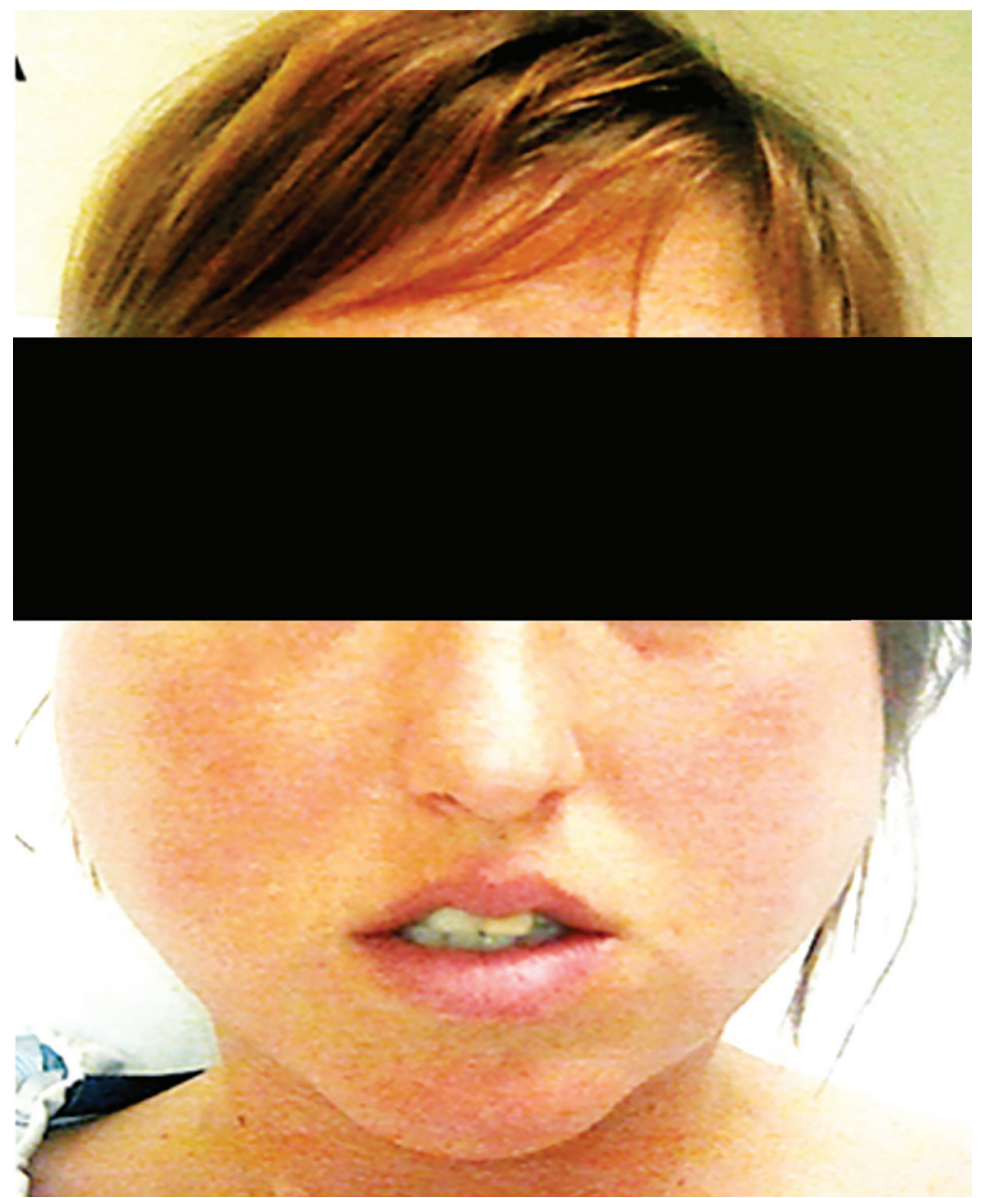

Figure 2. Sialadenosis.

ther cholinergic stimulation of the glands, hypertrophy of the glands to help meet demands of increased saliva production, or excessive backup of saliva that is no longer needed with cessation of vomiting.

Pathology study reveals hypertrophied acinar cells with otherwise preserved architecture without evidence of inflammation.

Swelling may subside with cessation of purging; failure of the parotid gland hypertrophy to resolve is highly suggestive of ongoing purging. ${ }^{18}$ Sialadenosis also tends to resolve with the use of sialagogues such as tart candies. Heating pads and nonsteroidal anti-inflammatory drugs also have a therapeutic role and perhaps should be prophylactically initiated in those with a long history of excessive vomiting who engage in treatment to stop purging. In rare refractory cases, pilocarpine may be judiciously used to reduce the glands back to normal size. ${ }^{19}$

\section{Cardiovascular}

The cardiac complications that are specific for purging include electrolyte disturbances as a result of vomiting and diuretic or laxative abuse. Conduction disturbances, including serious arrhythmias and QT prolongation, are increasingly encountered in those participating in various modes of purging due to the electrolyte disturbances that ensue, especially hypokalemia and acid-base disorders. Also, excessive ingestion of ipecac, which contains the cardiotoxic alkaloid emetine, to induce vomiting can lead to various conduction disturbances and potentially irreversible cardiomyopathy. ${ }^{20}$

Abuse of caffeine or stimulant medications used to treat attention-deficit/hyperactivity disorder may cause palpitations, sinus tachycardia, or cardiac arrhythmias such as supraventricular tachycardia. Similarly, diet pill abuse, which is increased in this population, is associated with arrhythmias. ${ }^{21}$

\section{Pulmonary}

Retching during vomiting increases intrathoracic and intra-alveolar pressures, which can lead to pneumomediastinum. ${ }^{22}$ Pneumomediastinum may also be encountered due to nontraumatic alveolar rupture in the setting of malnutrition and is therefore nonspecific in differentiating patients who purge from those who restrict. ${ }^{23}$ Vomiting also increases the risk of aspiration pneumonia. Aspiration may be involved in the heretofore enigmatic pathogenesis of pulmonary infection with Mycobacterium avium complex organisms.

\section{Gastrointestinal}

The gastrointestinal complications of purging depend on the mode of purging used. Upper gastrointestinal complications develop in those who engage in vomiting, whereas lower gastrointestinal complications develop in those who abuse stimulant laxatives.

Esophageal complications. Excessive vomiting exposes the esophagus to gastric acid and damages the lower esophageal sphincter, increasing the propensity for gastroesophageal reflux disease and other esophageal complications, including Barrett esophagus and esophageal adenocarcinoma. ${ }^{24}$ However, it is unclear if there truly is an association between purging by selfinduced vomiting and reflux disease. Although 
research indicates increased complaints of gastrointestinal reflux disease in those engaging in purging, and increased reflux may be present in those who purge when assessed by $\mathrm{pH}$ monitoring, endoscopic findings do not necessarily correlate with severity of reported symptoms. ${ }^{24,25}$ This suggests a possible functional component to gastrointestinal reflux-related concerns. ${ }^{26}$

Cessation of purging is the recommended treatment, although proton pump inhibitors can be tried. Metoclopramide may also be beneficial, given its actions of accelerating gastric emptying and increasing lower esophageal sphincter tone. Endoscopy should be considered if symptoms continue or have been present for many years, to look for the precancerous esophageal mucosal abnormalities found in Barrett esophagus.

Rare complications are esophageal rupture, known as Boerhaave syndrome, and MalloryWeiss tears, causing upper gastrointestinal bleeds due to the recurrent episodes of emesis. Mallory-Weiss tears commonly present as blood-streaked or tinged emesis or scant coffee ground emesis following recurrent vomiting episodes. Usually blood loss from such tears is minimal. Mallory-Weiss tears appear as longitudinal mucosal lacerations on endoscopy.

Colonic inertia. Individuals engaging in excessive and chronic stimulant laxative abuse may be at risk for "cathartic colon," a condition whereby the colon becomes an inert tube incapable of moving stool forward. This is believed due to direct damage to the gut myenteric nerve plexus. ${ }^{27}$ However, it is currently speculative as to whether this condition truly develops in those with eating disorders and with use of currently available stimulant laxatives. ${ }^{28}$ Regardless, in general, stimulant laxatives should be used only short-term, due to concerns regarding potential development of this condition, and should be stopped in those in whom it develops. Instead, osmotic laxatives, which do not directly stimulate peristalsis, are prescribed in a measured manner to manage constipation.

Melanosis coli, a black discoloration of the colon of no known clinical significance, is often reported during colonoscopy in those abusing stimulant laxatives. Rectal prolapse may also develop in those abusing stimulant laxatives, but again is nonspecific for this mode of purging as it can also develop solely as a consequence of malnutrition and the resultant weakness of the pelvic floor muscles.

\section{Endocrine}

A potential endocrine complication of $\mathrm{BN}$ is irregular menses ${ }^{29}$ as opposed to the amenorrhea frequently observed in both the restricting and binge-purge subtypes of anorexia nervosa.

Although patients with BN do not appear to be at a significantly increased risk for low bone mineral density-in contrast to those suffering from the restricting and binge-purge subtypes of anorexia nervosa- a bone density scan with dual-energy $\mathrm{x}$-ray absorptiometry may still be warranted to evaluate for bone disease in those with a past history of anorexia nervosa.

Patients with type 1 diabetes mellitus may manipulate their blood glucose levels as a means to purge calories, a condition previously referred to as diabulimia and now termed eating disorder-diabetes mellitus type $1 .{ }^{30}$ These patients are at risk of marked hyperglycemia, ketoacidosis, and premature microvascular complications such as retinopathy and neuropathy.

\section{METABOLIC AND ELECTROLYTE DISTURBANCES}

In addition to the above body system complications from purging, each of the common methods of purging used by patients with $\mathrm{BN}$ can be associated with specific electrolyte disturbances. These electrolyte abnormalities are likely the most proximate cause of death in patients with $\mathrm{BN}$. When a patient simultaneously engages in multiple modes of purging behaviors, just as their level of psychiatric illness can be more profound, so too the electrolyte disturbance profiles can overlap and be more extreme.

Patients with a history of a known purging behavior should be screened at increased frequency for serum electrolyte disturbances, up to even daily, depending on the frequency of their purging behaviors. ${ }^{31}$ In a study of patients admitted to inpatient and residential eating disorder treatment without prior medical stabilization, $26.2 \%$ of the $\mathrm{BN}$ patients presented with hypokalemia (potassium $<3.6$ $\mathrm{mmol} / \mathrm{L}$ ) on their admission laboratory test-
Purging by emesis increases the risk of aspiration pneumonia 


\section{TABLE 1}

\section{Summary of electrolyte disturbances in bulimia nervosa}

\begin{tabular}{llll} 
Behavior & Potassium & Sodium & Acid-base \\
\hline Self-induced vomiting & Low & Low or normal & Metabolic alkalosis \\
Laxative abuse & Low & Low or normal & Metabolic alkalosis or non-anion gap acidosis \\
Diuretic abuse & Low & Low or normal & Metabolic alkalosis
\end{tabular}

ing, while $8.5 \%$ had hyponatremia (sodium < $135 \mathrm{mmol} / \mathrm{L}$ ) and $23.4 \%$ had a metabolic alkalosis (bicarbonate $>28 \mathrm{mmol} / \mathrm{L}$ ). ${ }^{32}$

Self-induced vomiting is the most common method of purging in BN. ${ }^{33}$ Patients with selfinduced vomiting or diuretic abuse, or both, have been shown to present with hypokalemia, hypochloremia, and a metabolic alkalosis. ${ }^{34}$ The severity of the electrolyte abnormalities worsens with the frequency of vomiting.

Similarly, laxative abuse also results in hypokalemia and hypochloremia. However, either a non-anion gap metabolic acidosis or a metabolic alkalosis may be present, depending on the chronicity of the laxative abuse. ${ }^{35}$ Generally, more chronic diarrhea results in a metabolic alkalosis. Hyponatremia can also be present with these 3 purging behaviors. The hyponatremia encountered is most often of the hypovolemic type due to chronic fluid depletion as a result of the purging behaviors.

asymptomatic and can slowly be corrected

\section{Pathophysiology of hypokalemia and hypochloremia}

The pathophysiologic reasons for hypokalemia and hypochloremia seen with all significant purging behaviors are 2-fold and interrelated. First, and most obvious, there is loss of potassium in the purged gastric contents, excessive stool from laxative abuse, or in the urine through diuretic abuse.

Second, chronic purging results in intravascular fluid depletion. This fluid depletion is sensed by the afferent arteriole of the kidney as decreased renal perfusion pressure, which in turn activates the renin-angiotensin-aldosterone system, resulting in increased production of aldosterone by the zona glomerulosa of the adrenal glands. Aldosterone acts renally at the distal convoluted tubules and cortical collecting ducts, causing them to resorb sodium and chloride in the body's attempt to prevent severe dehydration, hypotension, and fainting. Aldosterone also promotes renal secretion of potassium into the urine and thus hypokalemia. This mechanism of potassium loss is actually a larger contributor to the hypokalemia than the actual gastrointestinal or urinary loses from the behaviors themselves.

The mechanisms by which metabolic alkalosis occurs in self-induced vomiting and in laxative abuse are similar. Initially, hydrogen ions and sodium chloride are lost in the vomitus or through diarrhea. The loss of hydrogen ions produces an alkalemic state. Intravascular volume depletion resulting from the loss of sodium chloride increases the resorption of bicarbonate within the proximal renal tubule, preventing its loss in the urine, which would normally occur to correct the alkalemia. Hypokalemia, if concurrently present, also increases bicarbonate resorption in the proximal tubule, further propagating the metabolic alkalosis. Lastly, increased serum aldosterone levels, brought about from intravascular volume depletion, fuel resorption of sodium at the expense of hydrogen and potassium, resulting in increased loss of hydrogen and potassium in the urine and further maintenance of the alkalemic state.

In diuretic abuse, the diuretics themselves act directly on the kidney to promote loss of sodium chloride in the urine, resulting in intravascular depletion and aldosterone secretion. This results in loss of hydrogen and potassium into the urine, resulting in a metabolic alkalosis. Potassium-sparing diuretics, such as spironolactone, however, do not precipitate a metabolic alkalosis, as they inhibit the action of aldosterone in the kidney.

Table 1 summarizes the electrolyte derangements that occur with $\mathrm{BN}$. 


\section{PSEUDO-BARTTER SYNDROME}

The aforementioned process of renin-angiotensin-aldosterone system activation results in what has been termed pseudo-Bartter syndrome due to resulting serum and histochemical findings on renal biopsy that resemble Bartter syndrome. ${ }^{36}$ However, the findings are not due to intrinsic renal pathology but rather are a result of the chronic state of dehydration from the purging behaviors. The resultant elevation in serum aldosterone, an integral part of pseudo-Bartter syndrome, can result in rapid edema formation when the purging behaviors are abruptly stopped. The reason is that the serum aldosterone levels remain high, causing salt and water retention even though the patient is no longer losing fluid, as the purging has ceased.

\section{EVALUATION AND MANAGEMENT OF ELECTROLYTE DISTURBANCES AND PSEUDO-BARTTER SYNDROME}

Covert purging should be strongly suspected in otherwise healthy young women presenting with hypokalemia without an alternative medical cause. ${ }^{37}$ However, hypokalemia alone is not specific for underlying purging behaviors. $^{34}$

If the patient is not forthcoming about their behavior when confronted, a spot urine potassium, creatinine, sodium, and chloride measurement can be obtained to further assess the source of potassium loss. A urine potassium-to-creatinine ratio less than 13 can identify hypokalemia resulting from gastrointestinal loss, diuretics, poor intake, or transcellular shifts. A urine sodium-to-chloride ratio can also be calculated. Vomiting is associated with a urine sodium-to-chloride ratio greater than 1.6 in the setting of hypokalemia, whereas laxative abuse is associated with a ratio less than 0.7.36

Chronic hypokalemia is often asymptomatic and can be corrected slowly. If the serum potassium level is no lower than $2.5 \mathrm{mmol} / \mathrm{L}$ and the patient has no physical symptoms or electrocardiographic changes of hypokalemia, the hypokalemia can be managed by stopping the purging behavior and giving oral potassium supplementation. ${ }^{38,39}$ Adherence to oral potassium repletion can be improved by using potassium chloride tablets rather than liquid preparations. ${ }^{38}$ Aggressive intravenous potassium supplementation places patients at risk of hyperkalemia and should be reserved for more critically low serum potassium levels.

Severe hypokalemia (serum potassium less than $2.5 \mathrm{mmol} / \mathrm{L}$ ) requires both oral and intravenous repletion of potassium. This repletion process is aided by giving isotonic saline with potassium chloride intravenously at a low infusion rate $(50-75 \mathrm{~mL} /$ hour $)$. Correcting the patient's volume depletion is required to correct the metabolic alkalosis and interrupt renin-angiotensin-aldosterone system activation. Untreated severe hypokalemia can result in a prolonged corrected QT interval on electrocardiography, subsequent torsades de pointes, and other life-threatening cardiac arrhythmias. Simply attempting to replete potassium without attention to the concomitant metabolic alkalosis will be unsuccessful because of the ongoing kaliuresis due to aldosterone's ongoing effects on the kidneys. Rarely, chronic hypokalemia has been associated with acute renal failure, with renal biopsy demonstrating interstitial nephritis, termed hypokalemic nephropathy. ${ }^{40}$

Mild hyponatremia often will autocorrect with interruption of purging behaviors and oral rehydration. However, if the serum sodium is less than $125 \mathrm{mmol} / \mathrm{L}$, hospitalization is warranted for close monitoring and for slow correction with isotonic saline-ie, at a rate that increases the serum sodium by no more than 4 to $6 \mathrm{mmol} / \mathrm{L}$ every 24 hours. This avoids the serious complication known as central pontine myelinolysis. ${ }^{41}$ If hyponatremia is severe (serum sodium $<118 \mathrm{mmol} / \mathrm{L}$ ), the patient will likely benefit from admission to an intensive care unit and renal consultation for consideration of administration of desmopressin to prevent overcorrection.

Metabolic alkalosis can develop in patients with $\mathrm{BN}$ as a result of decreased intravascular volume, elevated aldosterone, and hypokalemia; it is most often saline-responsive. A spot urine chloride can be used to inform care. If it is less than $10 \mathrm{mmol} / \mathrm{L}$, the metabolic alkalosis is hypovolemic and will improve with slow intravenous saline administration. Clinicians may also rely on physical examination to help determine the patient's volume status.

\section{Mild \\ hyponatremia will often correct itself with oral rehydration and interruption of purging behaviors}


Due to the underlying risk of pseudoBartter syndrome in patients with $\mathrm{BN}$ who abruptly stop purging, care should be taken to avoid aggressive fluid resuscitation. Interruption of purging behaviors in conjunction with rapid intravenous fluid resuscitation can result in marked and rapid edema formation and weight gain, which can be psychologically distressing. Thus, low infusion rates of saline $(50 \mathrm{~mL} /$ hour $)$ and low doses of spironolactone (50-100 mg initially with a maximum of 200-400 mg/day) should be initiated and titrated based on edema and weight trends to mitigate edema formation..$^{42}$ Spironolactone is generally continued for 2 to 4 weeks and then should be tapered by $50 \mathrm{mg}$ every few days thereafter. Occasionally, in extreme laxative abusers, proclivity toward edema may persist and necessitate an even slower spironolactone taper.

\section{MEDICAL COMPLICATIONS OF BINGE-EATING}

The literature on complications of bingeeating specific to $\mathrm{BN}$ is limited, and thus, we must look to studies of binge-eating disorder. However, patients with binge-eating disorder tend to be overweight or obese, as they do not purge after binge episodes. Thus, many of the medical complications in binge-eating disorder, such as type 2 diabetes, hypertension, nonalcoholic fatty liver disease, and metabolic syndrome are obesity-related. ${ }^{43}$

In contrast, many patients with $\mathrm{BN}$ have a normal body mass index. Therefore, it is difficult to infer that the medical complications that occur in binge-eating disorder are the same as those that occur from binge-eating in $\mathrm{BN}$. However, the extrapolation does make sense in some instances. For instance, patients who binge are at higher risk of nutritional deficiencies because food taken in during a binge tends to be processed, high in fat and carbohydrates, and low in protein. A diet low in vitamins, including $A$ and $C$, and minerals increases the risk of nutritional deficiencies. Additionally, patients with binge-eating disorder have more gastrointestinal complaints such as acid reflux, dysphagia, and bloating, which, as outlined above, are also seen in BN. Thus, bingeing may play a role in these symptoms.
Lastly, gastric perforation has been reported in patients with $\mathrm{BN}$ in the context of a bingeing episode marked by excessive stomach distention, resulting in gastric necrosis. ${ }^{44}$ Furthermore, gastric outlet obstruction has also been reported in this patient population due to formation of a food bezoar.

\section{IDENTIFICATION AND MENTAL HEALTH TREATMENT}

The Eating Disorder Screen for Primary Care has been shown to effectively screen patients for disordered eating in a general medicine setting. ${ }^{45}$ It consists of 5 questions:

- Are you satisfied with your eating pattern? ("No" is considered an abnormal response.)

- Do you ever eat in secret? ("Yes" is an abnormal response to this and the remaining questions.)

- Does your weight affect the way you feel about yourself?

- Have any members of your family suffered from an eating disorder?

- Do you currently suffer with or have you ever suffered in the past with an eating disorder?

Cotton et $\mathrm{al}^{45}$ found that an abnormal response to 2 or more of these questions had a sensitivity of $100 \%$ and a specificity of $71 \%$ for eating disorders.

Standard mental health treatments for BN include nutritional stabilization and behavior interruption, monitoring for and appropriate management of associated medical complications, prescribing medications as clinically indicated, and psychotherapeutic interventions. Cognitive behavioral therapy is the recommended initial intervention for the treatment of BN. A recent network meta-analysis suggested that guided cognitive behavioral selfhelp and a specific form of cognitive behavioral therapy-individual cognitive behavioral therapy for eating disorders-may most likely lead to full remission. ${ }^{46}$

No drug has been developed specifically for the treatment of BN (Table 2). Fluoxetine, with a target dose of $60 \mathrm{mg}$ daily independent of the presence of comorbidities, is the only medication approved by the US Food and Drug Administration for $\mathrm{BN}$. This selective 
serotonin reuptake inhibitor has been shown to reduce the frequency of binge-eating and purging episodes significantly, more so than fluoxetine $20 \mathrm{mg}$ daily and placebo. ${ }^{47}$ Fluoxetine is recommended for patients who do not respond adequately to psychotherapeutic interventions. ${ }^{48}$

Other selective serotonin reuptake inhibitor antidepressants along with the antiepileptic topiramate also have been shown to have modest efficacy. ${ }^{49}$ Bupropion, which has a boxed warning and is contraindicated in the treatment of BN, should not be used due to an increased risk of seizure.

No clinical trials have evaluated the use of stimulant medications in the treatment of BN. Often, stimulant medications are discontinued in patients until there is a period of abstinence from purging behaviors. Following abstinence, reinitiation of the stimulant could be reconsidered off-label if bingeing behaviors persist or attention deficit hyperactivity disorder is a comorbidity, or both. There can be some utility to reinitiation with a clear treatment agreement outlining expectations for maintaining efforts at purging symptom interruption and continued stimulant prescribing.

In general, concomitant treatment for anxiety or depression should be pursued if these co-occur with BN. Selective serotonin reuptake inhibitors such as fluoxetine would also target these symptoms. If a trial of fluoxetine has failed, then sertraline or escitalopram would be reasonable second-line options. Typically, citalopram would not be used due to higher risk of prolonged QT interval than other selective serotonin reuptake inhibitors, especially given the possibility of electrolyte abnormalities in BN. Paroxetine would not be used due to the potential for weight gain as a side effect.

\section{PROGNOSIS}

Increased risk of relapse has been associated with greater psychosocial dysfunction and body image disturbance. ${ }^{50}$ In patients requiring hospitalization, a number of factors have been shown to predict poor outcome, including fewer follow-up years, increased drive for thinness, older age at initial treatment, and more impairment in global functioning. ${ }^{50}$

\section{TABLE 2}

\section{Psychopharmacology clinical pearls}

Fluoxetine is the only US Food and Drug Administration-approved medication for the treatment of bulimia nervosa

Co-occurring anxiety and depression should be managed with therapy and pharmacologically

Stimulant medications have not been evaluated in the treatment of bulimia nervosa

Recovery is possible with variable remission rates, based on the type of study and definition of remission, from $38 \%$ and $42 \%$ at 11 and 21-year follow-up, respectively, and 65\% of individuals at a 9-year and 22-year followup. ${ }^{50,51}$ This reinforces the need to utilize accessible and effective treatments to achieve sustained recovery.

\section{CONCLUSION}

$\mathrm{BN}$ is a complex psychiatric disease with myriad medical complications, some of which may be life-threatening. Most of the morbidity and mortality in patients with $\mathrm{BN}$ is a direct result of the aforementioned purging behaviors and their resultant electrolyte and acid-base disorders. Thus, it is important that clinicians familiarize themselves with these complications as most patients with $\mathrm{BN}$ are of normal weight and are otherwise often able to avoid detection of their eating disorder.

\section{INITIAL CASE CONTINUED}

You release the patient in the initial clinical scenario from her follow-up appointment without intervention or follow-up laboratory testing. You fail to recognize the Russell sign, and you advise her that the edema is due to fluids administered in the emergency department and will self-resolve. She returns to her purging behaviors with increased vigor due to perceived weight gain from the edema.

One month later, she experiences a syncopal episode during cross-country practice, again necessitating oral potassium and intravenous saline administration. On follow-up, her edema is worse, and you recognize the Russell sign, having just read this review article. On follow-up laboratory testing, you note ongoing mild hypo-

\section{Bupropion is contraindicated in the treatment of bulimia nervosa due to an increased risk of seizure}


kalemia and screen her for an eating disorder. The screening is positive, and she discloses to you not only about her daily self-induced vomiting, but also her abuse of stimulant laxatives and bingeing episodes.

You initiate a referral to a residential treatment facility for eating disorders, start her on daily potassium chloride $40 \mathrm{mmol}$, and plan for weekly follow-up laboratory testing until she enters residential treatment.

Acknowledgments: The authors would like to thank Ms. Kelly Maebane for her superb assistance with formatting and editing of the manuscript.

\section{DISCLOSURES}

The authors report no relevant financial relationships which, in the context of their contributions, could be perceived as a potential conflict of interest.

\section{REFERENCES}

1. Arcelus J, Mitchell AJ, Wales J, Nielsen S. Mortality rates in patients with anorexia nervosa and other eating disorders. A metaanalysis of 36 studies. Arch Gen Psychiatry 2011; 68(7):724-731. doi:10.1001/archgenpsychiatry.2011.74

2. American Psychiatric Association (APA). Diagnostic and Statistical Manual of Mental Disorders. 5th ed. Washington, DC: American Psychiatric Association; 2013.

3. Mehler PS, Rylander M. Bulimia nervosa-medical complications. J Eat Disord 2015; 3:12. doi:10.1186/s40337-015-0044-4

4. Haedt AA, Edler C, Heatherton TF, Keel PK. Importance of multiple purging methods in the classification of eating disorder subtypes. Int J Eat Disord 2006; 39(8):648-654. doi:10.1002/eat.20335

5. Lichtenstein MB, Hinze CJ, Emborg B, Thomsen F, Hemmingsen SD. Compulsive exercise: links, risks and challenges faced. Psychol Res Behav Manag 2017; 10:85-95. doi:10.2147/PRBM.S113093

6. Kessler RC, Berglund PA, Chiu WT, et al. The prevalence and correlates of binge eating disorder in the World Health Organization World Mental Health Surveys. Biol Psychiatry 2013; 73(9):904-914. doi:10.1016/j.biopsych.2012.11.020

7. Feldman MB, Meyer IH. Eating disorders in diverse lesbian, gay, and bisexual populations. Int J Eat Disord 2007; 40(3):218-226. doi:10.1002/eat.20360

8. Wade TD. Recent research on bulimia nervosa. Psychiatr Clin North Am 2019; 42(1):21-32. doi:10.1016/j.psc.2018.10.002

9. Udo T, Grilo CM. Prevalence and correlates of DSM-5-defined eating disorders in a nationally representative sample of US adults. Biol Psychiatry 2018; 84(5):345-354. doi:10.1016/j.biopsych.2018.03.014

10. Caslini M, Bartoli F, Crocamo C, Dakanalis A, Clerici M, Carrà G. Disentangling the association between child abuse and eating disorders: a systematic review and meta-analysis. Psychosom Med 2016; 78(1):7990. doi:10.1097/PSY.0000000000000233

11. Himmerich $H$, Hotopf $M$, Shetty $H$, et al. Psychiatric comorbidity as a risk factor for the mortality of people with bulimia nervosa. Soc Psychiatry Psychiatr Epidemiol 2019; 54(7):813-821. doi:10.1007/s00127-019-01667-0

12. Cucchi A, Ryan D, Konstantakopoulos G, et al. Lifetime prevalence of non-suicidal self-injury in patients with eating disorders: a systematic review and meta-analysis. Psychol Med 2016; 46(7):1345-1358. doi:10.1017/S0033291716000027

13. Preti A, Rocchi MB, Sisti D, Camboni MV, Miotto P. A comprehensive meta-analysis of the risk of suicide in eating disorders. Acta Psychiatr Scand 2011; 124(1):6-17. doi:10.1111/j.1600-0447.2010.01641.x

14. Tith RM, Paradis G, Potter BJ, et al. Association of bulimia nervosa with long-term risk of cardiovascular disease and mortality among women. JAMA Psychiatry 2020; 77(1):44-51. doi:10.1001/jamapsychiatry.2019.2914

15. Strumia R. Eating disorders and the skin. Clin Dermatol 2013; 31(1):80-85. doi:10.1016/j.clindermatol.2011.11.011

16. Romanos GE, Javed F, Romanos EB, Williams RC. Oro-facial manifestations in patients with eating disorders. Appetite 2012; 59(2):499504. doi:10.1016/j.appet.2012.06.016

17. Garcia Garcia B, Dean Ferrer A, Diaz Jimenez N, Alamillos Granados FJ. Bilateral parotid sialadenosis associated with long-standing bulimia: a case report and literature review. J Maxillofac Oral Surg 2018;
17(2):117-121. doi:10.1007/s12663-016-0913-7

18. Vavrina J, Müller W, Gebbers JO. Enlargement of salivary glands in bulimia. J Laryngol Otol 1994; 108(6):516-518. doi:10.1017/s002221510012729x

19. Park KK, Tung RC, de Luzuriaga AR. Painful parotid hypertrophy with bulimia: a report of medical management. J Drugs Dermatol 2009; 8(6):577-579. pmid:19537384

20. Ho PC, Dweik R, Cohen MC. Rapidly reversible cardiomyopathy associated with chronic ipecac ingestion. Clin Cardiol 1998; 21(10):780783. doi: $10.1002 / \mathrm{clc} .4960211018$

21. Inayat F, Majeed CN, Ali NS, Hayat M, Vasim I. The risky side of weight-loss dietary supplements: disrupting arrhythmias causing sudden cardiac arrest. BMJ Case Rep 2018; 11(1):e227531. doi:10.1136/bcr-2018-227531

22. McCurdy JM, McKenzie CE, El-Mallakh RS. Recurrent subcutaneous emphysema as a consequence of bulimia nervosa. Int J Eat Disord 2013; 46(1):92-94. doi:10.1002/eat.22044

23. Jensen VM, Støving RK, Andersen PE. Anorexia nervosa with massive pulmonary air leak and extraordinary propagation. Int J Eat Disord 2017; 50(4):451-453. doi:10.1002/eat.22674

24. Denholm M, Jankowski J. Gastroesophageal reflux disease and bulimia nervosa-a review of the literature. Dis Esophagus 2011; 24(2):79-85. doi:10.1111/j.1442-2050.2010.01096.x

25. Kiss A, Wiesnagrotzki S, Abatzi TA, Meryn S, Haubenstock A, Base W. Upper gastrointestinal endoscopy findings in patients with longstanding bulimia nervosa. Gastrointest Endosc 1989; 35(6):516-518. doi:10.1016/s0016-5107(89)72901-1

26. Abraham S, Kellow JE. Do the digestive tract symptoms in eating disorder patients represent functional gastrointestinal disorders? BMC Gastroenterol 2013; 13:38. doi:10.1186/1471-230X-13-38

27. Smith B. Pathology of cathartic colon. Proc R Soc Med 1972; 65(3):288. pmid:5083323

28. Müller-Lissner S. What has happened to the cathartic colon? Gut 1996; 39(3):486-488. doi:10.1136/gut.39.3.486

29. Gendall KA, Bulik CM, Joyce PR, Mclntosh VV, Carter FA. Menstrual cycle irregularity in bulimia nervosa. Associated factors and changes with treatment. J Psychosom Res 2000; 49(6):409-415. doi:10.1016/s0022-3999(00)00188-4

30. Deiana V, Diana E, Pinna F, et al. Clinical features in insulin-treated diabetes with comorbid diabulimia, disordered eating behaviors and eating disorders. Eur Psychiatry 2016; 33:S81.

31. Edler C, Haedt AA, Keel PK. The use of multiple purging methods as an indicator of eating disorder severity. Int J Eat Disord 2007; 40(6):515-520. doi:10.1002/eat.20416

32. Mehler PS, Blalock DV, Walden $\mathrm{K}$, et al. Medical findings in 1,026 consecutive adult inpatient-residential eating disordered patients. Int J Eat Disord 2018; 51(4):305-313. doi:10.1002/eat.22830

33. Mitchell JE, Hatsukami D, Eckert ED, Pyle RL. Characteristics of 275 patients with bulimia. Am J Psychiatry 1985; 142(4):482-485. doi:10.1176/ajp.142.4.482

34. Wolfe BE, Metzger ED, Levine JM, Jimerson DC. Laboratory screening for electrolyte abnormalities and anemia in bulimia nervosa: a controlled study. Int J Eat Disord 2001; 30(3):288-293. doi:10.1002/eat.1086

35. Mehler PS, Walsh K. Electrolyte and acid-base abnormalities associated with purging behaviors. Int J Eat Disord 2016; 49(3):311-318. doi:10.1002/eat.22503 


\section{NITSCH AND COLLEAGUES}

36. Bahia A, Mascolo M, Gaudiani JL, Mehler PS. PseudoBartter syndrome in eating disorders. Int J Eat Disord 2012; 45(1):150-153. doi:10.1002/eat.20906

37. Wu KL, Cheng CJ, Sung CC, et al. Identification of the causes for chronic hypokalemia: importance of urinary sodium and chloride excretion. Am J Med 2017; 130(7):846-855. doi:10.1016/j.amjmed.2017.01.023

38. Cohn JN, Kowey PR, Whelton PK, Prisant LM. New guidelines for potassium replacement in clinical practice: a contemporary review by the National Council on Potassium in Clinical Practice. Arch Intern Med 2000; 160(16):2429-2436. doi:10.1001/archinte.160.16.2429

39. Gennari FJ. Hypokalemia. N Engl J Med 1998; 339(7):451-458. doi:10.1056/NEJM199808133390707

40. Menahem SA, Perry GJ, Dowling J, Thomson NM. Hypokalaemia-induced acute renal failure. Nephrol Dial Transplant 1999; 14(9):22162218. doi:10.1093/ndt/14.9.2216

41. Sterns RH. Disorders of plasma sodium - causes, consequences, and correction. N Engl J Med 2015; 372(1):55-65. doi:10.1056/NEJMra1404489

42. Brown CA, Mehler PS. Successful "detoxing" from commonly utilized modes of purging in bulimia nervosa. Eat Disord 2012; 20(4):312-320. doi:10.1080/10640266.2012.689213

43. Wassenaar E, Friedman J, Mehler PS. Medical complications of binge eating disorder. Psychiatr Clin North Am 2019; 42(2):275-286. doi:10.1016/j.psc.2019.01.010

44. Bern EM, Woods ER, Rodriguez L. Gastrointestinal manifestations of eating disorders. J Pediatr Gastroenterol Nutr 2016; 63(5):e77-e85. doi:10.1097/MPG.0000000000001394
45. Cotton MA, Ball C, Robinson P. Four simple questions can help screen for eating disorders. J Gen Intern Med 2003; 18(1):53-56. doi:10.1046/j.1525-1497.2003.20374.x

46. Svaldi J, Schmitz F, Baur J, et al. Efficacy of psychotherapies and pharmacotherapies for bulimia nervosa. Psychol Med 2019; 49(6):898-910. doi:10.1017/S0033291718003525

47. Goldstein DJ, Wilson MG, Thompson VL, Potvin JH, Rampey AH Jr. Long-term fluoxetine treatment of bulimia nervosa. Fluoxetine Bulimia Nervosa Research Group. Br J Psychiatry 1995; 166(5):660-666. doi:10.1192/bjp.166.5.660

48. Walsh BT, Agras WS, Devlin MJ, et al. Fluoxetine for bulimia nervosa following poor response to psychotherapy. Am J Psychiatry 2000; 157(8):1332-1334. doi:10.1176/appi.ajp.157.8.1332

49. McElroy SL, Guerdjikova Al, Mori N, Romo-Nava F. Progress in developing pharmacologic agents to treat bulimia nervosa. CNS Drugs 2019; 33(1):31-46. doi:10.1007/s40263-018-0594-5

50. Quadflieg N, Fichter MM. Long-term outcome of inpatients with bulimia nervosa-results from the Christina Barz Study. Int J Eat Disord 2019; 52(7):834-845. doi:10.1002/eat.23084

51. Eddy KT, Tabri N, Thomas JJ, et al. Recovery from anorexia nervosa and bulimia nervosa at 22-year follow-up. J Clin Psychiatry 2017; 78(2):184-189. doi:10.4088/JCP.15m10393

Address: Philip S. Mehler, MD, FACP, FAED, Denver Health Medical Center, 723 Delaware Street, Pav M, Denver, CO 80204; pmehler@dhha.org 